\title{
Performance of Using Porous Floating Cylindrical Breakwater with Different Porosities as a Wave Energy Dissipater in Experimental Study
}

\author{
Y. M. El Saie \\ Associate Professor, Head of Civil Engineering Department. \\ Higher Institute of Engineering El Shorouk City, Cairo, Egypt
}

\begin{abstract}
Floating breakwaters are commonly used to protect shorelines, marine structures and harbors from wave attacks. Through the physical model test method, the wave energy dissipation performance by using porous floating cylindrical breakwater under regular waves. The objective of this research is to study the effect of using a horizontal cylindrical pipe with different diameters and different percentage of porosities acting as a wave energy dissipater, working as a single pipe or as a group. Which contains five different models with diameters ( 2 inch, 3 inch, 4 inch, (3\&4 inch together) and $(2,3 \&$ 4 inch together), by choosing different percentage of porosities as $(15,30 \& 50 \%)$ to achieve maximum energy dissipation with minimum transmitted energy. The experiments were carried out in a recirculating open channel flume located at the Hydraulics Engineering Laboratory, Higher Institute of Engineering in El Shorouk City.
\end{abstract}

\section{INTRODUCTION}

As a wave resisting structure, breakwaters are widely used in port fairways, bathing places, wharves a yachts, and its structural form has great influence on ship navigation, water exchange, wave attenuation and wave reduction in offshore engineering. Traditional gravity breakwaters in deep-water areas are faced with problems such as high foundation requirements, difficult construction and high cost in very deep water. The floating breakwater is made up of floating bodies and mooring systems, which owns a lot of advantages. It is conducive to the water exchange, and has little influence on the sediment and the ecological environment. The cost is less affected by water depth, the foundation is more adaptable, and the construction is relatively convenient. It is more suitable for sea areas with high tidal range, soft soil bed and deep waters. Therefore, the floating breakwaters has broad application prospects. In recent years, many researchers proposed many new types of floating bodies, the cage floating breakwater, the square box-truss-mooring-type floating breakwater, the double pontoon floatingbreakwater, the net-board floating breakwater, the wave dissipation performance, hydrodynamic performance, the mooring force and wave pressure of floating breakwaters are mainly studied in experimental and numerical ways. Many experimental and theoretical studies have been done for determining the efficiency of vertical submerged or emerged permeable or impermeable types of breakwaters. Dalrymple et al, (1991) examined the reflection and the transmission coefficients from porous structures under oblique wave attack. Wang and Sun, (2010) examined a porous breakwater where the structure was fabricated with large numbers of diamond-shaped blocks arranged to reduce transmitted wave height and the mooring force. Their results showed that the porous FB reduced transmission of a large part of the incident wave energy through dissipation rather than reflection of the wave energy. The effect of the porous media on the incoming waves has in recent years gained attention with the use of advanced numerical models as discussed for example by Garcia et al., (2004), Jacobsen et al., (2015), Jensen et al., (2014), Losada et al., (2005). The single 
pontoon FB reflects rather than dissipates the wave energy, Abul-Azm, (1993) analyzed the linear wave potential near submerged thin barriers using the Eigen Function Expansion to determine the breakwater efficiency. Erik et al., (2018), attaching wing plates to the floating breakwater significantly reduced the motion, which was also anticipated. When the porous sides were attached the motion of the Floating Breakwater increased compared to the (WP) cross section, but the wave transmission was reduced. Isaacson et al, (1996), carried out an experimental investigation on the reflection of obliquely incident waves from a model rubblemound breakwater of single slice. N Zheng et al., (2018), the wave dissipation performance and mooring force of porous floating breakwater under regular waves are analyzed and compared ith the traditional pontoon floating breakwater. Heikal and Attar, (1997), examined the efficiency of an impermeable, vertical thin submerged breakwater sited on sloping impermeable bed experimentally and numerically by using the Eigen Function Expansion method Twu et al, (2001), studied theoretically, using the Eigen Function Expansion method, the problem of wave transmission over a rectangular and vertically stratified with multi-slice porous material. Zhichao et al., (2018), study of the wave-dissipating performance of a fourlayer horizontal porous-plate breakwater. Stamos and Hajj, (2001) developed a new method to separate incident and reflected wave components. Then the method is applied to perform a parametric study to compare the reflection and transmission characteristics of flexible and rigid breakwaters. Stamos et al, (2003) conducted a parametric experimental study to compare the reflection and transmission characteristics of submerged hemi-cylindrical and rectangular rigid and water-filled flexible breakwater models. Ting et al, (2004) investigated how the porosity of submerged breakwaters affects non-breaking wave transformations. Eight model geometries each with six different porosities, from 0.421 to 0.912 , were also considered. Sidek and Wahab, (2007) experimentally investigated the effects of porosity of submerged breakwater on non-breaking wave transformations. A hollow framework-shaped test model was used. Three models each with three different porosities ranging from 0.40 to 0.80 were used in that experiment. Womera, (2011) investigated the interaction between waves and rectangular submerged impermeable breakwater. A two-dimensional numerical model based on the SOLA-VOF method was proposed. Vahid and Fatemeh, (2011) developed an experimental solution for evaluation of transmission coefficients of water waves through single perforated sheets and upright perforated wave filters. Wu et al, (2012) conducted experimental and numerical interactions. The Effect of Porosity of Submerged and Emerged Breakwater on wave transmission. From the review of many related published literature in this field, it is acknowledged that more information are needed from both in the laboratory and the field for the efficient application, development and design of promising vertical porous structures as coastal engineering solutions in the field. In this study, experimental investigation has been carried out in a two dimensional wave tank to understand the effect of porosity in vertical thick submerged and emerged porous breakwaters under wave action. In summary, through the research of many floating breakwaters, the wave dissipation performance of floating breakwater under long wave action is the focus. In order to increase the effects on long waves, some percentage of porosities in the body of the floating cylindrical breakwater by opening holes in it on both sides of the model to dissipate more wave energy. It is also be called porous floating cylindrical breakwater. In this paper, the transmission and dissipation coefficients can be calculated and also choosing the favorite percentage of porosity and the best diameter for achieving minimum transmitted energy.

\section{EXPERIMENTAL WORK}

The experimental work was conducted in a wave flume $12.0 \mathrm{~m} \mathrm{long}, 50 \mathrm{~cm}$ wide and $60 \mathrm{~cm}$ deep. The side walls are glass panels. The flume is provided with a wave generator. 
The tests were carried out in a wave flume in the Hydraulic laboratory at the Higher Institute of Engineering, in El Shorouk City. The flume is $12.00 \mathrm{~m}$ long, $0.50 \mathrm{~m}$ wide, and the sidewalls are $0.60 \mathrm{~m}$ high, see Fig. (1). The sidewalls of the flume consisted of a 12.00 panels of glass each one meter, to enable for following the motion of the Floating Breakwater as shown in figure (2). The flume was equipped with a piston-type wave maker at one end and a wave absorber before it and a wave absorber at the other side of the flume. The water depth in the flume was fixed to $30 \mathrm{~cm}$, as a maximum water depth in the flume.

Wave period and wave length are measured by Sonic Wave Sensor XB as shown in figure (3).

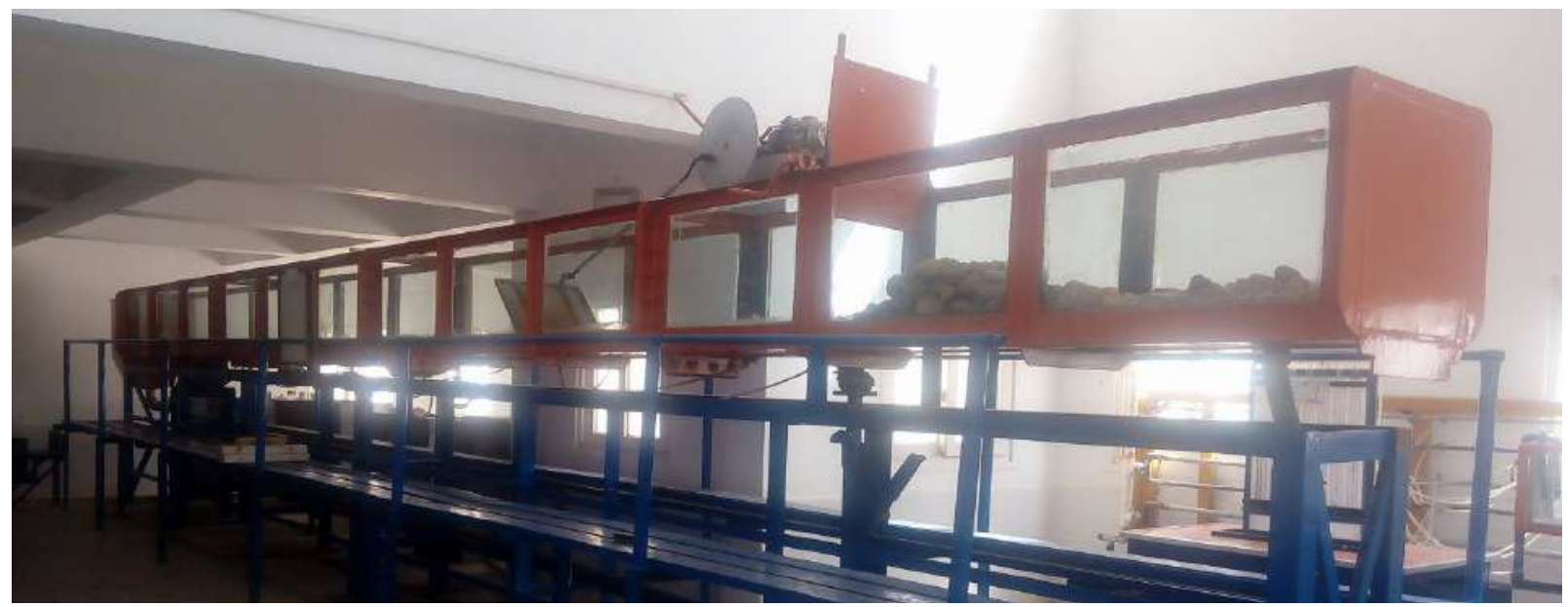

Figure (1) General view of the wave flume

\section{DESIGN OF THE PHYSICAL MODELS}

The physical model consists of five models of a hollow cylindrical horizontal floating pipes with different diameters (2", 3", 4", (3" \& 4" together) and (2", 3"\& 4" together), changing the porosity from (15, 30 and $50 \%$ ), it rested on a wooden floating base with dimensions of $45 \mathrm{~cm}$ long, $16 \mathrm{~cm}$ width and $7 \mathrm{~cm}$ height, which tied to the base of the flume by four anchors. The horizontal pipe hanging over two vertical sides of height $14 \mathrm{~cm}$. Water depth was constant at $30 \mathrm{~cm}$ ) with different wave heights, lengths and periods. By testing these models to evaluate the performance of each model for dissipate the incident wave energy and also to calculate wave coefficients ( $\mathrm{Ct}, \mathrm{Cr}$ and $\mathrm{Cd}$ ), also the maximum possible transmitted energy. Finally, comparison these tested models by a same solid pipes, El saie, (2019). Also the figures $(2,3,4$, $5,6,7 \& 8$ ) contains some of the tested models with different porosities.

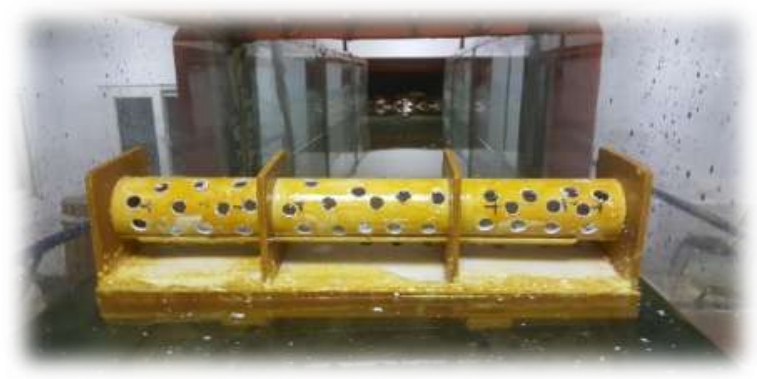

Fig. (2) Model one with $P=30 \%$

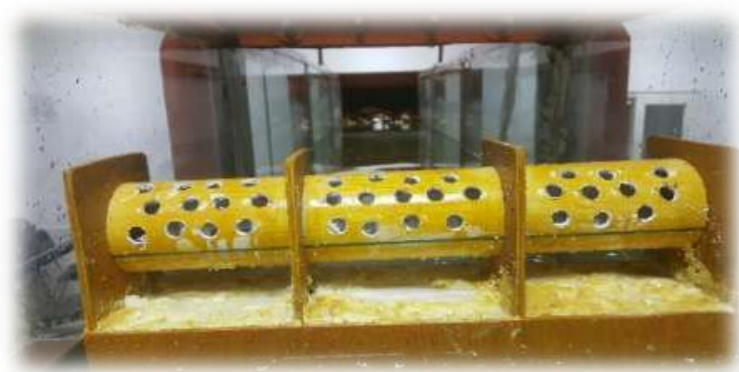

Fig. (3) Model two with $P=30 \%$ 


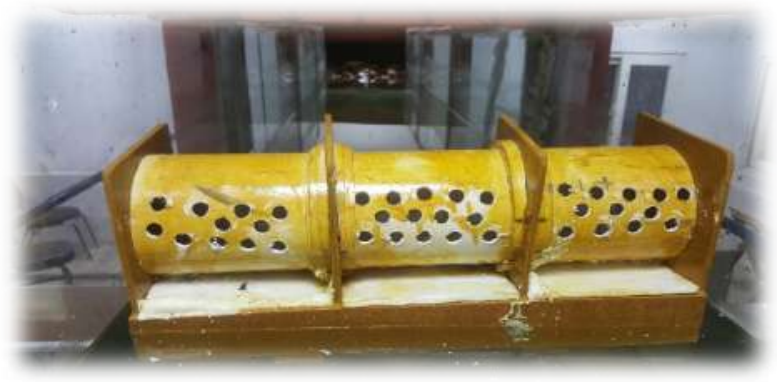

Fig. (4) Model three with $P=50 \%$

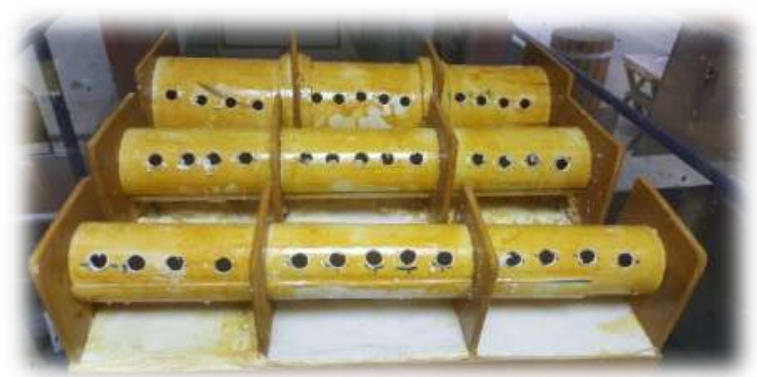

Fig. (6) Model five with $P=15 \%$

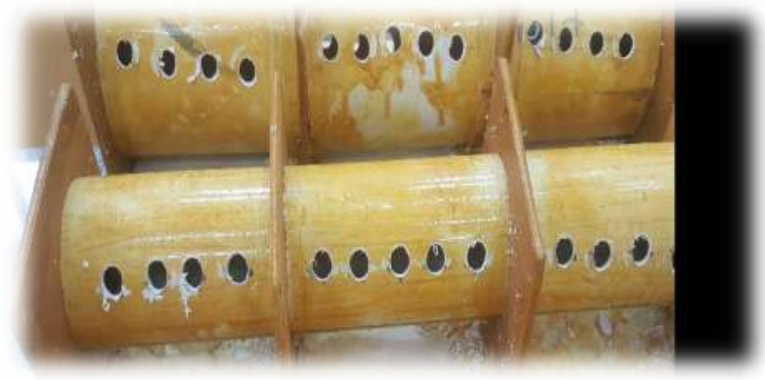

Fig. (5) Model four with $P=15 \%$

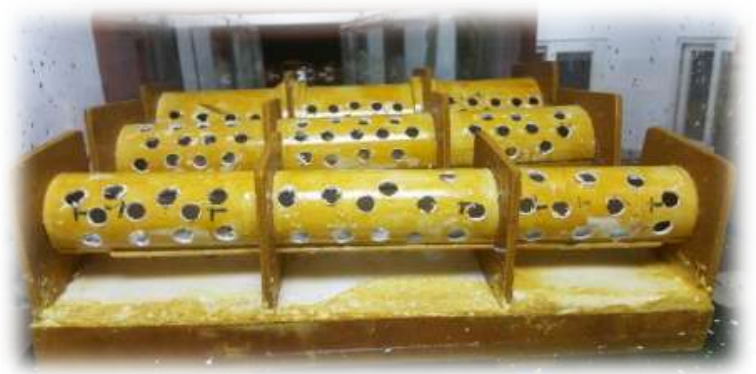

Fig. (7) Model five with $P=30 \%$

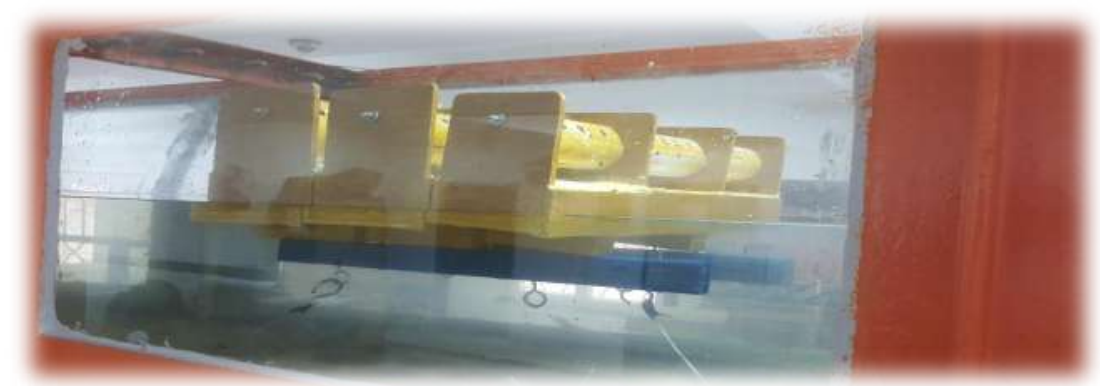

Fig. (8) Side view of model five tied with anchors

\section{MEASUREMENTS AND RESULTS}

By testing these models in the wave flume and measure the wave heights before and after these tested models by using Sonic Wave Sensor XB, measurements and calculated coefficients ( $\mathrm{Ct}, \mathrm{Cr} \& \mathrm{Cd}$ ) as shown in the following tables.

Tables $(1,2, \& 3)$ for $\emptyset=2$ inch $\& P=15,30 \& 50 \%$.

Table (1) Measured and calculated data for $\emptyset=2$ inch $\& P=15 \%$

\begin{tabular}{|c|c|c|c|c|c|c|c|c|c|}
\hline \multirow{6}{*}{ 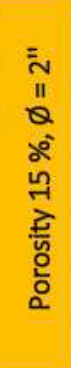 } & $\begin{array}{c}\mathrm{Hi} \\
\text { (cm) }\end{array}$ & $\begin{array}{c}\text { Ht } \\
\text { (cm) }\end{array}$ & $\begin{array}{c}\mathbf{H r} \\
(\mathrm{cm})\end{array}$ & $\begin{array}{l}\text { Length } \\
\text { (cm) }\end{array}$ & $\mathbf{H i} / \mathbf{L}$ & Ct & $\mathrm{Cr}$ & Cd & $\begin{array}{c}\% \text { energy } \\
\text { transmitted }\end{array}$ \\
\hline & 10.06 & 9.04 & 0.21 & 139.37 & 0.072 & 0.8989 & 0.0209 & 0.4376 & 80.80 \\
\hline & 9.01 & 7.90 & 0.20 & 122.34 & 0.074 & 0.8767 & 0.0219 & 0.4805 & 76.87 \\
\hline & 7.96 & 6.89 & 0.18 & 106.55 & 0.075 & 0.8660 & 0.0231 & 0.4996 & 74.99 \\
\hline & 7.34 & 5.99 & 0.17 & 90.23 & 0.081 & 0.8154 & 0.0227 & 0.5784 & 66.50 \\
\hline & 6.15 & 4.82 & 0.15 & 69.25 & 0.089 & 0.7844 & 0.0242 & 0.6198 & 61.52 \\
\hline
\end{tabular}


Table (2) Measured and calculated data for $\emptyset=2$ inch $\& P=30 \%$

\begin{tabular}{|c|c|c|c|c|c|c|c|c|c|}
\hline \multirow{6}{*}{ 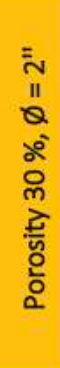 } & $\begin{array}{c}\mathbf{H i} \\
(\mathrm{cm})\end{array}$ & $\begin{array}{c}\mathrm{Ht} \\
\text { (cm) }\end{array}$ & $\begin{array}{c}\mathrm{Hr} \\
\text { (cm) }\end{array}$ & $\begin{array}{c}\text { Length } \\
\text { (cm) }\end{array}$ & $\mathbf{H i} / \mathbf{L}$ & Ct & $\mathrm{Cr}$ & Cd & $\begin{array}{l}\% \text { energy } \\
\text { transmitted }\end{array}$ \\
\hline & 10.06 & 9.27 & 0.18 & 139.37 & 0.072 & 0.9216 & 0.0183 & 0.3876 & 84.94 \\
\hline & 9.01 & 8.13 & 0.17 & 122.339 & 0.074 & 0.9018 & 0.0191 & 0.4317 & 81.33 \\
\hline & 7.96 & 7.20 & 0.16 & 106.548 & 0.075 & 0.9044 & 0.0202 & 0.4263 & 81.79 \\
\hline & 7.34 & 6.24 & 0.15 & 90.231 & 0.081 & 0.8495 & 0.0198 & 0.5272 & 72.17 \\
\hline & 6.15 & 5.10 & 0.13 & 69.254 & 0.089 & 0.8294 & 0.0212 & 0.5583 & 68.78 \\
\hline
\end{tabular}

Table (3) Measured and calculated data for $\emptyset=2$ inch $\& P=50 \%$

\begin{tabular}{|c|c|c|c|c|c|c|c|c|c|}
\hline \multirow{5}{*}{ 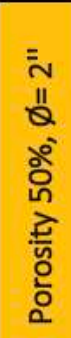 } & $\begin{array}{c}\mathrm{Hi} \\
(\mathrm{cm})\end{array}$ & $\begin{array}{c}\text { Htt } \\
(\mathrm{cm})\end{array}$ & $\begin{array}{c}\mathrm{Hr} \\
(\mathrm{cm})\end{array}$ & $\begin{array}{l}\text { Length } \\
\text { (cm) }\end{array}$ & Hi/L & Ct & $\mathrm{Cr}$ & Cd & $\begin{array}{l}\text { \% energy } \\
\text { transmitted }\end{array}$ \\
\hline & 10.06 & 9.52 & 0.14 & 139.37 & 0.072 & 0.9467 & 0.0138 & 0.3218 & 89.63 \\
\hline & 9.01 & 8.33 & 0.13 & 122.339 & 0.074 & 0.9240 & 0.0144 & 0.3822 & 85.37 \\
\hline & 7.96 & 7.33 & 0.12 & 106.548 & 0.075 & 0.9203 & 0.0152 & 0.3910 & 84.69 \\
\hline & 7.34 & 6.33 & 0.11 & 90.231 & 0.081 & 0.8618 & 0.0149 & 0.5070 & 74.27 \\
\hline & 6.15 & 5.20 & 0.10 & 69.254 & 0.089 & 0.8453 & 0.0160 & 0.5341 & 71.45 \\
\hline
\end{tabular}

Therefor figure (9) describe the relation between coefficient of dissipation (Cd) and (Hi/L) for $\emptyset=2.0$ inch with different percentage of porosity.

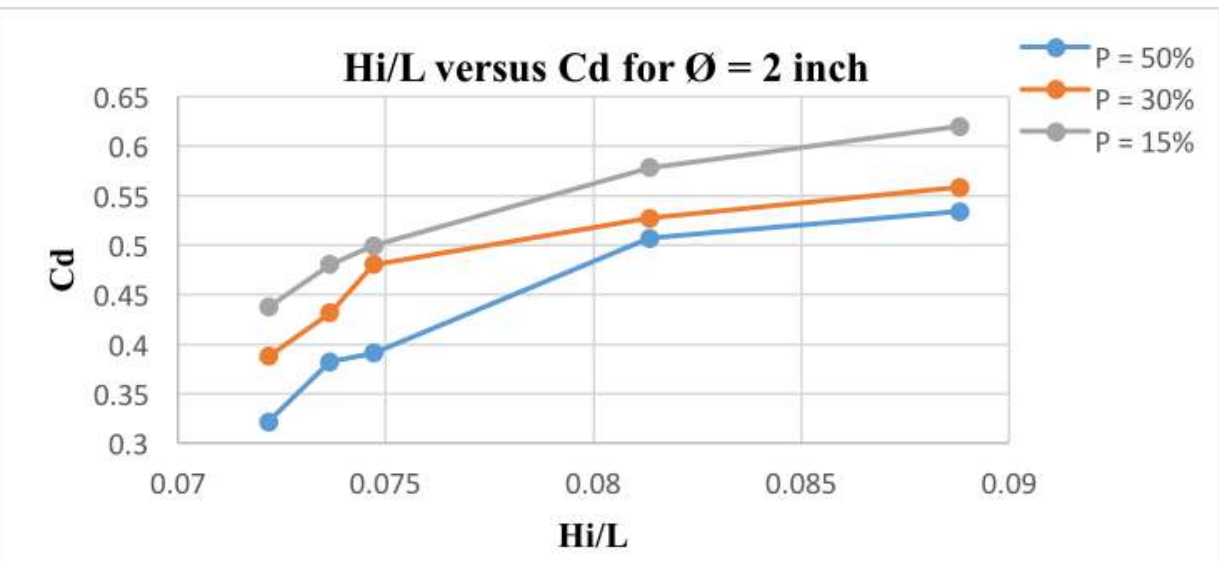

Fig. (9) Cd versus Hi/L for $\emptyset=2.0$ inch $\& P=(15,30 \& 50 \%)$

Also, figure (10) shows the percentage of energy transmitted with different wave steepness $\mathrm{Hi} / \mathrm{L}$ for $\emptyset=3.0$ inch and different porosities. 


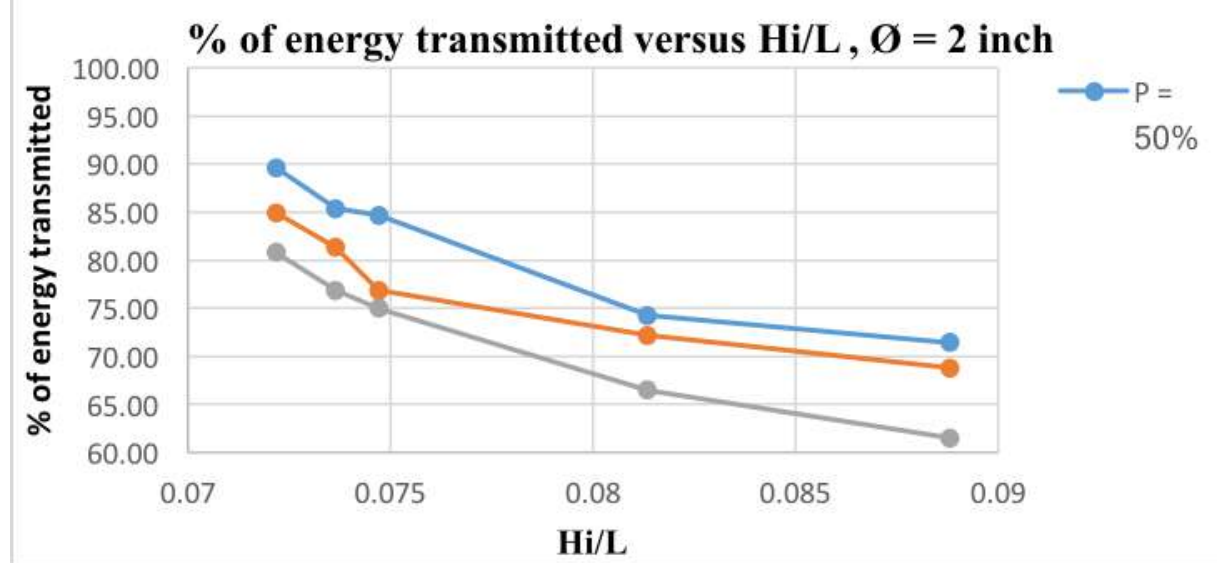

Fig. (10) \% of energy transmitted versus $\mathrm{Hi} / \mathrm{L}$ for $\emptyset=2.0$ inch $\& P=(15,30 \& 50 \%)$

Therefore, tables $(4,5 \& 6)$ also for measured heights of waves and the calculated wave coefficients for $\emptyset=3$ inch \& $P=15,30 \& 50 \%$.

Table (4) Measured and calculated data for $\emptyset=3$ inch $\& P=15 \%$

\begin{tabular}{|c|c|c|c|c|c|c|c|c|c|}
\hline \multirow{6}{*}{ 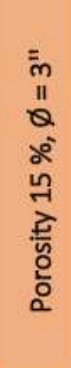 } & $\begin{array}{c}\mathrm{Hi} \\
(\mathrm{cm})\end{array}$ & $\begin{array}{c}\text { Ht } \\
(\mathrm{cm})\end{array}$ & $\begin{array}{c}\mathrm{Hr} \\
(\mathrm{cm})\end{array}$ & $\begin{array}{c}\text { Length } \\
\text { (cm) }\end{array}$ & $\mathbf{H i} / \mathbf{L}$ & Ct & $\mathbf{C r}$ & Cd & $\begin{array}{l}\text { \% energy } \\
\text { transmitted }\end{array}$ \\
\hline & 10.06 & 8.93 & 0.23 & 139.37 & 0.072 & 0.8880 & 0.0227 & 0.4594 & 78.8465 \\
\hline & 9.01 & 7.83 & 0.22 & 122.34 & 0.074 & 0.8685 & 0.0239 & 0.4950 & 75.4370 \\
\hline & 7.96 & 6.76 & 0.20 & 106.55 & 0.075 & 0.8495 & 0.0253 & 0.5269 & 72.1690 \\
\hline & 7.34 & 6.04 & 0.18 & 90.23 & 0.081 & 0.8235 & 0.0250 & 0.5668 & 67.8125 \\
\hline & 6.15 & 4.88 & 0.17 & 69.25 & 0.089 & 0.7932 & 0.0270 & 0.6083 & 62.9194 \\
\hline
\end{tabular}

Table (5) Measured and calculated data for $\emptyset=3$ inch $\& P=30 \%$

\begin{tabular}{|c|c|c|c|c|c|c|c|c|c|}
\hline \multirow{6}{*}{ 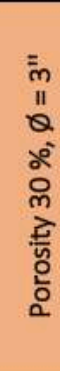 } & $\begin{array}{c}\mathrm{Hi} \\
(\mathrm{cm})\end{array}$ & $\begin{array}{c}\text { Ht } \\
(\mathrm{cm})\end{array}$ & $\begin{array}{c}\mathrm{Hr} \\
(\mathrm{cm})\end{array}$ & $\begin{array}{c}\text { Length } \\
\text { (cm) }\end{array}$ & $\mathrm{Hi} / \mathrm{L}$ & $\mathbf{C t}$ & $\mathrm{Cr}$ & Cd & $\begin{array}{c}\% \text { energy } \\
\text { transmitted }\end{array}$ \\
\hline & 10.06 & 9.3721 & 0.23 & 139.37 & 0.072 & 0.9316 & 0.0225 & 0.3627 & 86.79 \\
\hline & 9.01 & 8.1256 & 0.21 & 122.339 & 0.074 & 0.9018 & 0.0237 & 0.4314 & 81.33 \\
\hline & 7.96 & 7.1255 & 0.20 & 106.548 & 0.075 & 0.8952 & 0.0251 & 0.4450 & 80.13 \\
\hline & 7.34 & 6.2459 & 0.18 & 90.231 & 0.081 & 0.8509 & 0.0248 & 0.5247 & 72.41 \\
\hline & 6.15 & 5.0254 & 0.16 & 69.254 & 0.089 & 0.8171 & 0.0267 & 0.5758 & 66.77 \\
\hline
\end{tabular}

Table (6) Measured and calculated data for $\emptyset=3$ inch $\& P=50 \%$

\begin{tabular}{|c|c|c|c|c|c|c|c|c|c|}
\hline \multirow{6}{*}{ 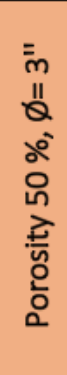 } & $\begin{array}{c}\mathbf{H i} \\
(\mathrm{cm})\end{array}$ & $\begin{array}{c}\text { Ht } \\
(\mathrm{cm})\end{array}$ & $\begin{array}{c}\mathrm{Hr} \\
(\mathrm{cm})\end{array}$ & $\begin{array}{c}\text { Length } \\
(\mathrm{cm})\end{array}$ & $\mathrm{Hi} / \mathrm{L}$ & $\mathbf{C t}$ & $\mathrm{Cr}$ & Cd & $\begin{array}{c}\% \text { energy } \\
\text { transmitted }\end{array}$ \\
\hline & 10.06 & 9.84 & 0.20 & 7 & 0.072 & 0.9777 & 0.0200 & 0.2092 & .58 \\
\hline & 9.01 & 8.62 & 0.19 & 122.339 & 0.074 & 0.9563 & 0.0210 & 0.2917 & 91.45 \\
\hline & 7.96 & 7.45 & 0.18 & 106.548 & 0.075 & 0.9353 & 0.0223 & 0.3530 & 87.49 \\
\hline & 7.34 & 6.66 & 0.16 & 90.231 & 0.081 & 0.9067 & 0.0220 & 0.4213 & 82.21 \\
\hline & 6.15 & 5.37 & 0.15 & 69.254 & 0.089 & 0.8734 & 0.0237 & 0.4865 & 76.27 \\
\hline
\end{tabular}

Therefor figure (11) describe the relation between coefficient of dissipation (Cd) and (Hi/L) for $\varnothing=3.0$ inch with different percentage of porosity. 


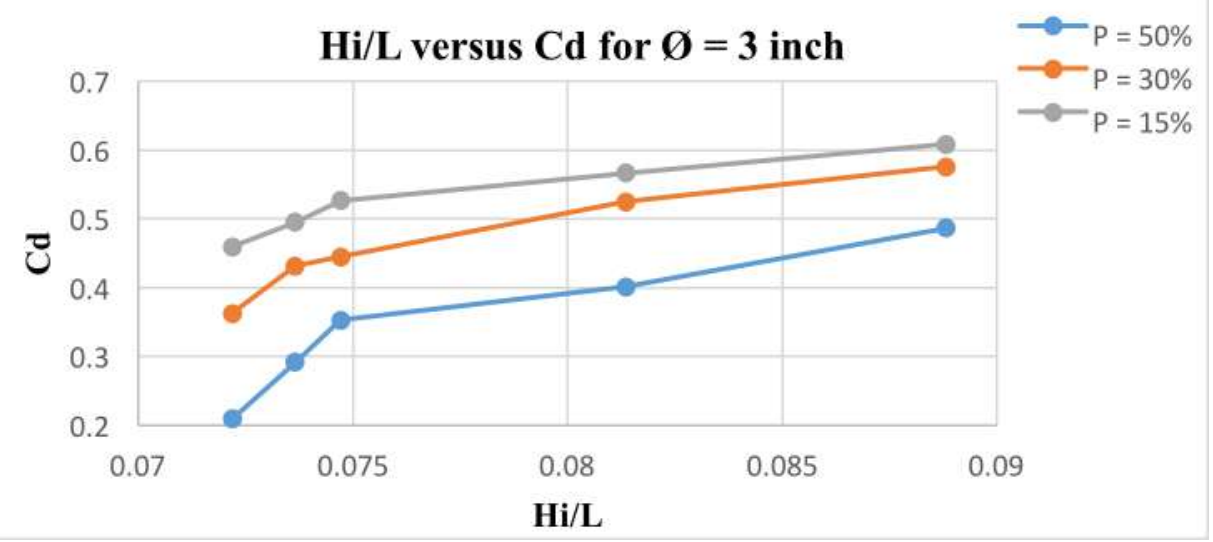

Fig. (11) Cd versus $\mathrm{Hi} / \mathrm{L}$ for $\emptyset=3.0$ inch $\& \mathrm{P}=(15,30 \& 50 \%)$

Also, figure (12) shows the percentage of energy transmitted with different wave steepness $\mathrm{Hi} / \mathrm{L}$ for $\emptyset=3.0$ inch and different porosities.

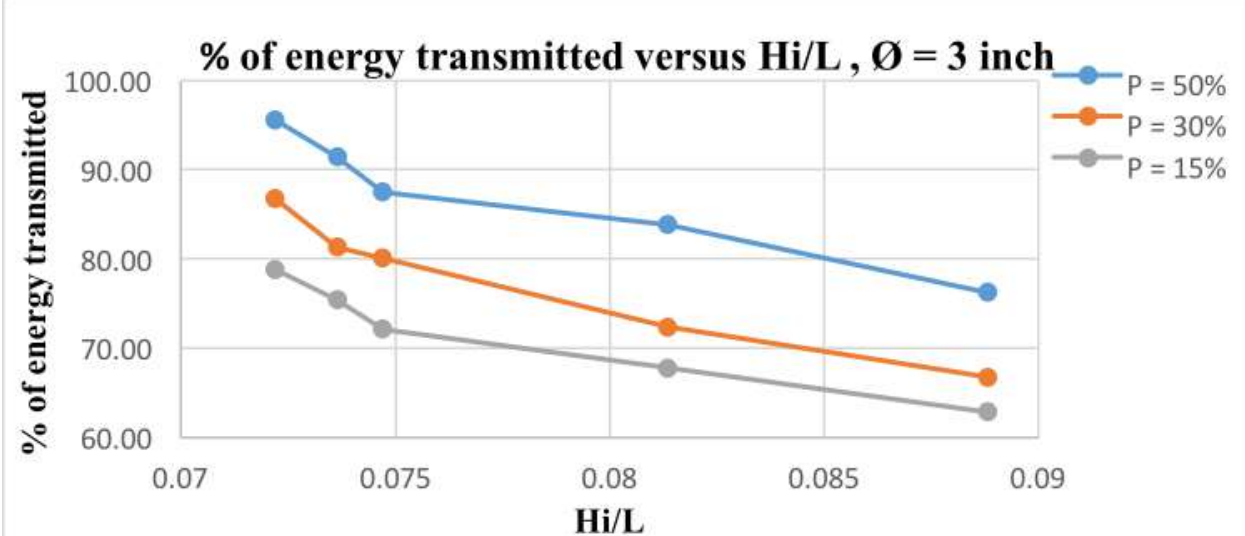

Fig. (12) \% of energy transmitted versus $\mathrm{Hi} / \mathrm{L}$ for $\emptyset=3.0$ inch $\& P=(15,30 \& 50 \%)$

Similarly, tables (7, 8 \&9) also for measured heights of waves and the calculated wave coefficients for $\emptyset=4$ inch \& $P=15,30 \& 50 \%$.

Table (7) Measured and calculated data for $\emptyset=4$ inch $\& P=15 \%$

\begin{tabular}{|c|c|c|c|c|c|c|c|c|c|}
\hline \multirow{6}{*}{ 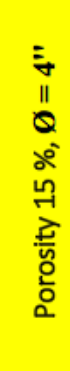 } & $\begin{array}{c}\mathbf{H i} \\
(\mathbf{c m})\end{array}$ & $\begin{array}{c}\text { Ht } \\
\text { (cm) }\end{array}$ & $\begin{array}{c}\mathrm{Hr} \\
(\mathrm{cm})\end{array}$ & $\begin{array}{c}\text { Length } \\
\text { (cm) }\end{array}$ & $\mathbf{H i} / \mathbf{L}$ & $\mathbf{C t}$ & $\mathbf{C r}$ & Cd & $\begin{array}{c}\% \text { energy } \\
\text { transmitted }\end{array}$ \\
\hline & 10.06 & 8.766 & 0.22 & 139.37 & 0.072 & 0.8714 & 0.0219 & 0.4901 & 75.93 \\
\hline & 9.01 & 7.644 & 0.21 & 122.339 & 0.074 & 0.8484 & 0.0231 & 0.5289 & 71.97 \\
\hline & 7.96 & 6.625 & 0.20 & 106.548 & 0.075 & 0.8322 & 0.0246 & 0.5539 & 69.26 \\
\hline & 7.34 & 5.714 & 0.18 & 90.231 & 0.081 & 0.7784 & 0.0244 & 0.6273 & 60.59 \\
\hline & 6.15 & 4.457 & 0.16 & 69.254 & 0.089 & 0.7247 & 0.0264 & 0.6885 & 52.52 \\
\hline
\end{tabular}


Table (8) Measured and calculated data for $\emptyset=4$ inch $\& P=30 \%$

\begin{tabular}{|c|c|c|c|c|c|c|c|c|c|}
\hline \multirow{6}{*}{ 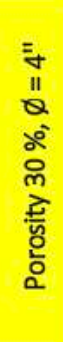 } & $\begin{array}{c}\mathbf{H i} \\
(\mathbf{c m})\end{array}$ & $\begin{array}{c}\mathrm{Ht} \\
(\mathbf{c m})\end{array}$ & $\begin{array}{c}\mathrm{Hr} \\
\text { (cm) }\end{array}$ & $\begin{array}{c}\text { Length } \\
\text { (cm) }\end{array}$ & Hi/L & Ct & $\mathrm{Cr}$ & Cd & $\begin{array}{l}\text { \% energy } \\
\text { transmitted }\end{array}$ \\
\hline & 10.06 & 9.1888 & 0.21 & 139.37 & 0.072 & 0.9134 & 0.0210 & 0.4065 & 83.43 \\
\hline & 9.01 & 8.0124 & 0.20 & 122.339 & 0.074 & 0.8893 & 0.0221 & 0.4568 & 79.08 \\
\hline & 7.96 & 6.8458 & 0.19 & 106.548 & 0.075 & 0.8724 & 0.0235 & 0.4883 & 73.96 \\
\hline & 7.34 & 6.1025 & 0.17 & 90.231 & 0.081 & 0.8159 & 0.0234 & 0.5777 & 69.12 \\
\hline & 6.15 & 4.672 & 0.16 & 69.254 & 0.089 & 0.7597 & 0.0253 & 0.6498 & 57.71 \\
\hline
\end{tabular}

Table (9) Measured and calculated data for $\emptyset=4$ inch \& $P=50 \%$

\begin{tabular}{|c|c|c|c|c|c|c|c|c|c|}
\hline \multirow{6}{*}{ 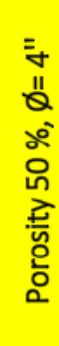 } & $\begin{array}{c}\mathbf{H i} \\
(\mathrm{cm}) \\
\end{array}$ & $\begin{array}{c}\mathbf{H t} \\
(\mathrm{cm}) \\
\end{array}$ & $\begin{array}{c}\mathbf{H r} \\
(\mathrm{cm}) \\
\end{array}$ & $\begin{array}{c}\text { Length } \\
\text { (cm) }\end{array}$ & $\mathrm{Hi} / \mathrm{L}$ & $\mathbf{C t}$ & $\mathrm{Cr}$ & Cd & $\begin{array}{c}\% \text { energy } \\
\text { transmitted }\end{array}$ \\
\hline & 10.06 & 9.64 & 0.18 & 139.37 & 0.072 & 0.9586 & 0.0180 & 0.2840 & 91.90 \\
\hline & 9.01 & 8.32 & 0.17 & 122.339 & 0.074 & 0.9333 & 0.0190 & 0.3585 & 85.36 \\
\hline & 7.96 & 7.29 & 0.16 & 106.548 & 0.075 & 0.9156 & 0.0202 & 0.4017 & 83.83 \\
\hline & 7.34 & 6.29 & 0.15 & 90.231 & 0.081 & 0.8564 & 0.0200 & 0.5160 & 73.34 \\
\hline & 6.15 & 4.82 & 0.13 & 69.254 & 0.089 & 0.7973 & 0.0217 & 0.6032 & 61.52 \\
\hline
\end{tabular}

Therefore, figure (13) describe the relation between coefficient of dissipation (Cd) and (Hi/L) for $\varnothing=4.0$ inch with different percentage of porosities.

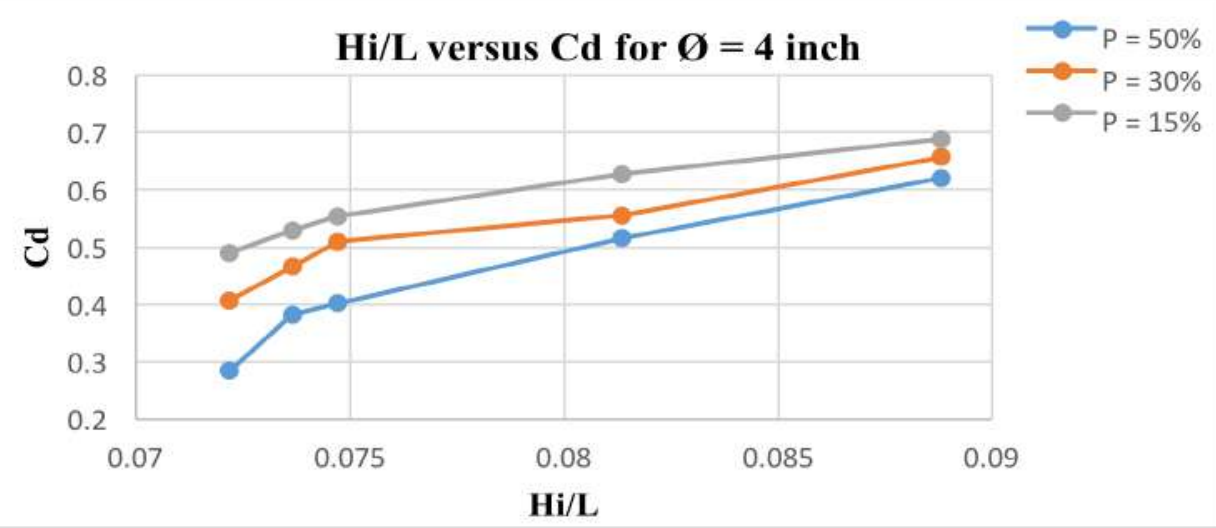

Fig. (13) Cd versus $\mathrm{Hi} / \mathrm{L}$ for $\emptyset=4.0$ inch $\& P=(15,30 \& 50 \%)$

Also, figure (14) shows the percentage of energy transmitted with different wave steepness $\mathrm{Hi} / \mathrm{L}$ for $\emptyset=4.0$ inch and different porosities.

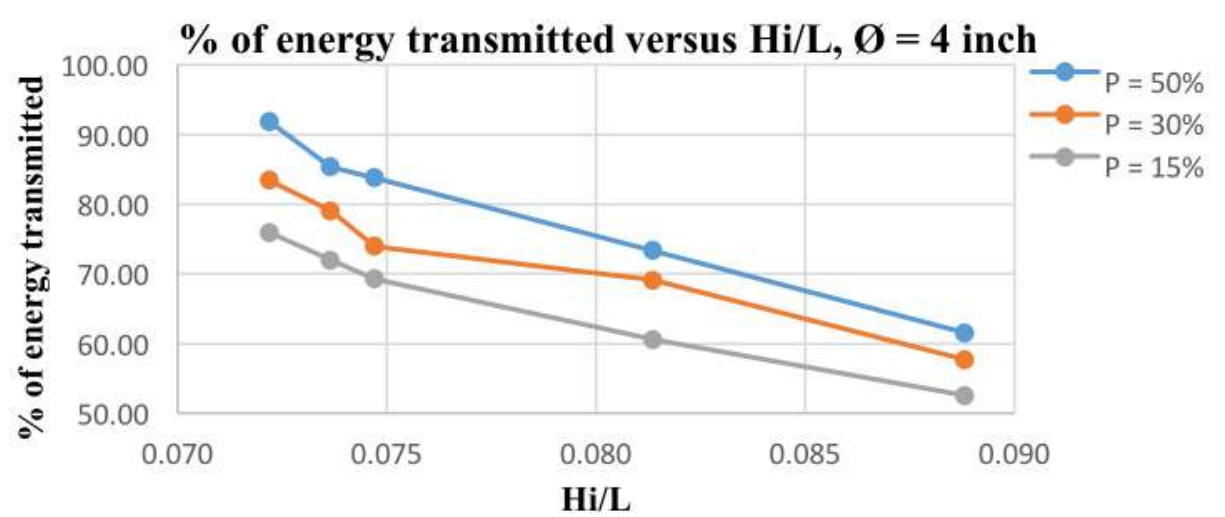

Fig. (14) \% of energy transmitted versus $\mathrm{Hi} / \mathrm{L}$ for $\emptyset=4.0$ inch $\& P=(15,30 \& 50 \%)$ 
Therefore, tables $(10,11 \& 12)$ also for measured wave heights and the calculated wave coefficients for $\emptyset=3 \& 4$ inch $\& P=15,30 \& 50 \%$.

Table (10) Measured and calculated data for $\emptyset=3 \& 4$ inch $\& P=15 \%$

\begin{tabular}{|c|c|c|c|c|c|c|c|c|c|}
\hline \multirow{6}{*}{ 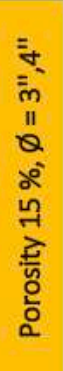 } & $\begin{array}{c}\mathrm{Hi} \\
(\mathrm{cm})\end{array}$ & $\begin{array}{c}\mathrm{Ht} \\
(\mathrm{cm}) \\
\end{array}$ & $\begin{array}{c}\mathrm{Hr} \\
(\mathrm{cm})\end{array}$ & $\begin{array}{c}\text { Length } \\
(\mathrm{cm})\end{array}$ & $\mathrm{Hi} / \mathrm{L}$ & $\mathbf{C t}$ & $\mathrm{Cr}$ & Cd & $\begin{array}{c}\% \text { energy } \\
\text { transmitted }\end{array}$ \\
\hline & 10.06 & 5.607 & 0.26 & 139.37 & 0.072 & 0.5573 & 0.0263 & 0.8299 & 31.06 \\
\hline & 9.01 & 4.89 & 0.25 & 122.339 & 0.074 & 0.5562 & 0.0278 & 0.8306 & 29.53 \\
\hline & 7.96 & 4.192 & 0.24 & 106.548 & 0.075 & 0.5267 & 0.0297 & 0.8496 & 27.74 \\
\hline & 7.34 & 3.716 & 0.22 & 90.231 & 0.081 & 0.5063 & 0.0297 & 0.8619 & 25.63 \\
\hline & 6.15 & 2.91 & 0.20 & 69.254 & 0.089 & 0.5064 & 0.0325 & 0.8617 & 22.43 \\
\hline
\end{tabular}

Table (11) Measured and calculated data for $\emptyset=3 \& 4$ inch $\& P=30 \%$

\begin{tabular}{|c|c|c|c|c|c|c|c|c|c|}
\hline \multirow{6}{*}{ 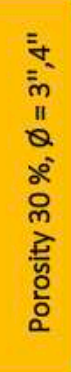 } & $\begin{array}{c}\mathbf{H i} \\
(\mathrm{cm})\end{array}$ & $\begin{array}{c}\text { Ht } \\
(\mathrm{cm})\end{array}$ & $\begin{array}{c}\mathrm{Hr} \\
(\mathrm{cm})\end{array}$ & $\begin{array}{c}\text { Length } \\
\text { (cm) }\end{array}$ & $\mathrm{Hi} / \mathrm{L}$ & Ct & $\mathbf{C r}$ & d & $\begin{array}{c}\% \\
\text { tran }\end{array}$ \\
\hline & 10.06 & 5.8721 & 0.25 & 139.37 & 0.072 & 0.5837 & 0.0249 & 0.8116 & 34.07 \\
\hline & 9.01 & 5.2032 & 0.24 & 122 & 0.074 & 0.5825 & 0.0263 & 0.8124 & 33.35 \\
\hline & 7.96 & 4.3907 & 0.22 & 106.548 & 0.075 & 0.5516 & 0.0282 & 0.8336 & 30.43 \\
\hline & 7.34 & 3.892 & 0.21 & 90.231 & 0.081 & 0.5302 & 0.0282 & 0.8474 & 28.12 \\
\hline & 6.15 & 3.03 & 0.19 & 69.254 & 0.089 & 0.5304 & 0.0308 & 0.8472 & 24.18 \\
\hline
\end{tabular}

Table (12) Measured and calculated data for $\emptyset=3 \& 4$ inch $\& P=50 \%$

\begin{tabular}{|c|c|c|c|c|c|c|c|c|c|}
\hline \multirow{6}{*}{ 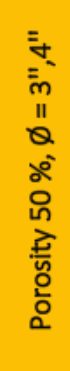 } & $\begin{array}{c}\mathbf{H i} \\
(\mathrm{cm})\end{array}$ & $\begin{array}{c}\mathbf{H t} \\
(\mathrm{cm})\end{array}$ & $\begin{array}{c}\mathrm{Hr} \\
(\mathrm{cm})\end{array}$ & $\begin{array}{c}\text { Length } \\
(\mathrm{cm})\end{array}$ & $\mathrm{Hi} / \mathrm{L}$ & $\mathbf{C t}$ & $\mathrm{Cr}$ & Cd & $\begin{array}{c}\% \text { energy } \\
\text { transmitted }\end{array}$ \\
\hline & 10.06 & 6.16 & 0.21 & 139.37 & 0.072 & 0.6122 & 0.0213 & 0.7904 & 37.47 \\
\hline & 9.01 & 5.29 & 0.20 & 122.339 & 0.074 & 0.6109 & 0.0226 & 0.7914 & 34.61 \\
\hline & 7.96 & 4.66 & 0.19 & 106.548 & 0.075 & 0.5785 & 0.0241 & 0.8153 & 34.27 \\
\hline & 7.34 & 4.08 & 0.18 & 90.231 & 0.081 & 0.5561 & 0.0241 & 0.8308 & 30.92 \\
\hline & 6.15 & 3.21 & 0.16 & 69.254 & 0.089 & 0.5562 & 0.0263 & 0.8306 & 27.29 \\
\hline
\end{tabular}

Also, figure (15) describe the relation between coefficient of dissipation (Cd) and (Hi/L) for $\emptyset=3.0 \& 4.0$ inch with different percentage of porosities.

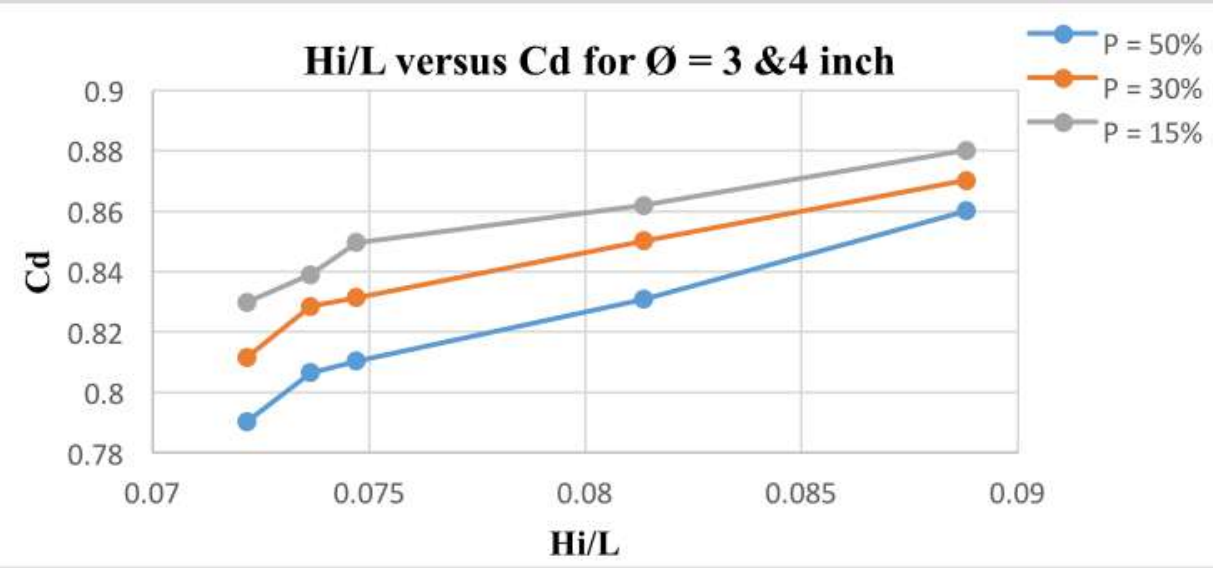

Fig. (15) Cd versus $\mathrm{Hi} / \mathrm{L}$ for $\emptyset=4.0$ inch $\& P=(15,30 \& 50 \%)$ 
Therefore, figure (16) shows the percentage of energy transmitted with different wave steepness $\mathrm{Hi} / \mathrm{L}$ for $\emptyset=3.0 \& 4.0$ inch and different porosities.

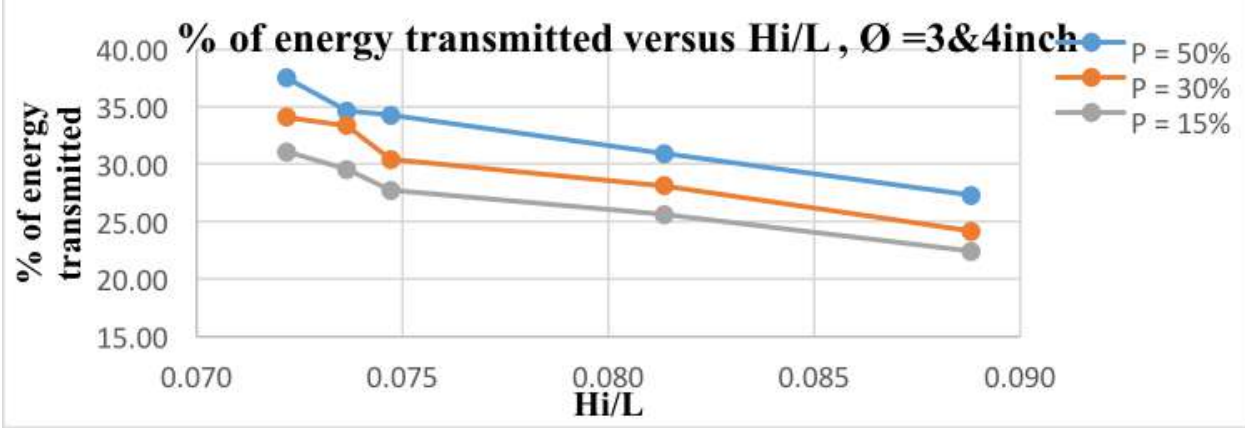

Fig. (16) \% of energy transmitted versus $\mathrm{Hi} / \mathrm{L}$ for $\emptyset=3.0 \& 4.0$ inch $\& P=(15,30 \& 50 \%)$

Similarly, tables $(13,14 \& 15)$ also for measured heights of waves and the calculated wave coefficients for $\emptyset=3.0,4.0 \& 4.0$ inch $\& P=15,30 \& 50 \%$.

Table (13) Measured and calculated data for $\emptyset=2.0,3.0 \& 4.0$ inch $\& P=15 \%$

\begin{tabular}{|c|c|c|c|c|c|c|c|c|c|}
\hline \multirow{6}{*}{ 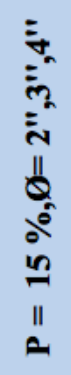 } & $\begin{array}{c}\mathbf{H i} \\
(\mathrm{cm})\end{array}$ & $\begin{array}{c}\mathbf{H t} \\
(\mathrm{cm})\end{array}$ & $\begin{array}{c}\mathrm{Hr} \\
(\mathrm{cm})\end{array}$ & $\begin{array}{c}\text { Length } \\
\text { (cm) }\end{array}$ & $\mathrm{Hi} / \mathbf{L}$ & $\mathbf{C t}$ & $\mathrm{Cr}$ & Cd & \\
\hline & 10.06 & 4.976 & 0.29 & 139 & 0.072 & 0.4947 & 0.029 & 0.8686 & 24.47 \\
\hline & 9.01 & 4.201 & 0.28 & 339 & 0.074 & 0.4731 & 0.031 & 0.8805 & 1.71 \\
\hline & 7.96 & 3.672 & 0.27 & 106.548 & 0.075 & 0.4613 & 0.033 & 0.8866 & 21.28 \\
\hline & 7.34 & 3.294 & 0.25 & 90.231 & 0.081 & 0.4487 & 0.034 & 0.8930 & 20.14 \\
\hline & 6.15 & 2.703 & 0.23 & 69.254 & 0.089 & 0.4395 & 0.037 & 0.8975 & 19.31 \\
\hline
\end{tabular}

Table (14) Measured and calculated data for $\emptyset=2.0,3.0 \& 4.0$ inch $\& P=30 \%$

\begin{tabular}{|c|c|c|c|c|c|c|c|c|c|}
\hline \multirow{6}{*}{ 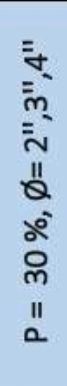 } & $\begin{array}{c}\mathbf{H i} \\
(\mathrm{cm})\end{array}$ & $\begin{array}{c}\text { Ht } \\
(\mathrm{cm})\end{array}$ & $\begin{array}{c}\mathrm{Hr} \\
(\mathrm{cm})\end{array}$ & $\begin{array}{c}\text { Length } \\
(\mathrm{cm})\end{array}$ & $\mathbf{H i} / \mathbf{L}$ & $\mathbf{C t}$ & $\mathbf{C r}$ & Cd & $\begin{array}{l}\% \text { energy } \\
\text { transmitted }\end{array}$ \\
\hline & 10.06 & 5.1518 & 0.27 & 139.37 & 0.072 & 0.5121 & 0.0267 & 0.8585 & 26.23 \\
\hline & 9.01 & 4.4132 & 0.26 & 122.339 & 0.074 & 0.4898 & 0.0284 & 0.8714 & 23.99 \\
\hline & 7.96 & 3.8014 & 0.24 & 106.548 & 0.075 & 0.4776 & 0.0306 & 0.8781 & 22.81 \\
\hline & 7.34 & 3.368 & 0.23 & 90.231 & 0.081 & 0.4646 & 0.0309 & 0.8850 & 21.06 \\
\hline & 6.15 & 2.798 & 0.21 & 69.254 & 0.089 & 0.4550 & 0.0341 & 0.8899 & 20.70 \\
\hline
\end{tabular}

Table (15) Measured and calculated data for $\emptyset=2.0,3.0 \& 4.0$ inch $\& P=50 \%$

\begin{tabular}{|c|c|c|c|c|c|c|c|c|c|}
\hline \multirow{6}{*}{ 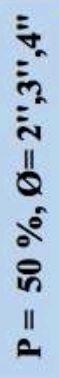 } & $\begin{array}{c}\mathrm{Hi} \\
(\mathrm{cm})\end{array}$ & $\begin{array}{c}\mathrm{Ht} \\
(\mathrm{cm})\end{array}$ & $\begin{array}{c}\mathrm{Hr} \\
(\mathrm{cm})\end{array}$ & $\begin{array}{c}\text { Length } \\
(\mathrm{cm})\end{array}$ & $\mathrm{Hi} / \mathrm{L}$ & $\mathbf{C t}$ & $\mathrm{Cr}$ & Cd & $\begin{array}{c}\% \text { energy } \\
\text { transmitted }\end{array}$ \\
\hline & 10.06 & 5.36 & 0.23 & 139.37 & 0.072 & 0.5325 & 0.0228 & 0.8462 & 28.35 \\
\hline & 9.01 & 4.65 & 0.22 & 122.339 & 0.074 & 0.5093 & 0.0243 & 0.8603 & 26.73 \\
\hline & 7.96 & 4.01 & 0.21 & 106.548 & 0.075 & 0.4965 & 0.0262 & 0.8676 & 25.41 \\
\hline & 7.34 & 3.55 & 0.19 & 90.231 & 0.081 & 0.4830 & 0.0264 & 0.8752 & 23.33 \\
\hline & 6.15 & 2.91 & 0.18 & 69.254 & 0.089 & 0.4730 & 0.0292 & 0.8806 & 22.38 \\
\hline
\end{tabular}

Also, figure (17) describe the relation between coefficient of dissipation (Cd) and (Hi/L) for $\emptyset=2.0,3.0 \& 4.0$ inch with different percentage of porosities. 


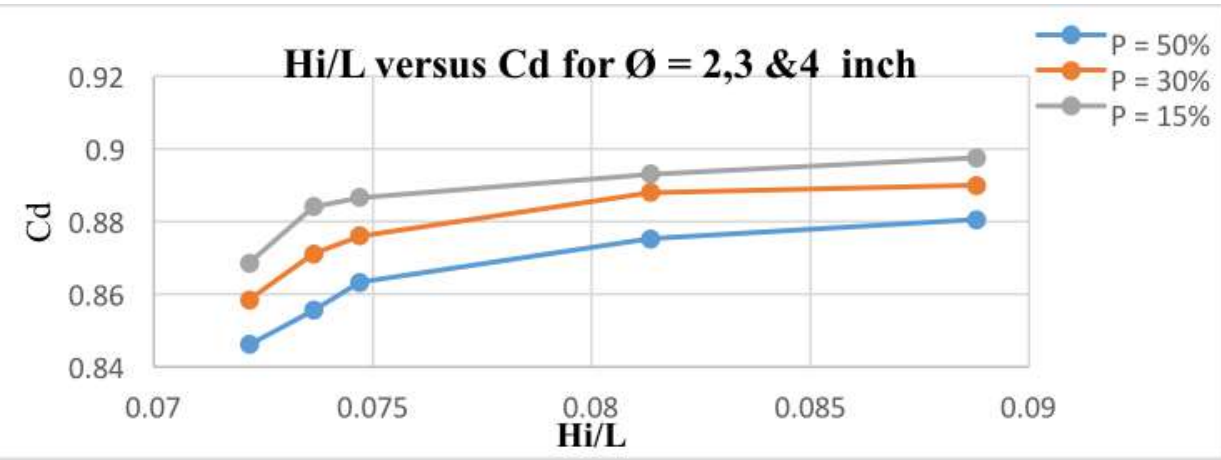

Fig. (17) Cd versus $\mathrm{Hi} / \mathrm{L}$ for $\emptyset=2.0,3.0 \& 4.0$ inch $\& P=(15,30 \& 50 \%)$

Therefore, figure (18) shows the percentage of energy transmitted with different wave steepness $\mathrm{Hi} / \mathrm{L}$ for $\emptyset=2.0,3.0 \& 4.0$ inch and different porosities.

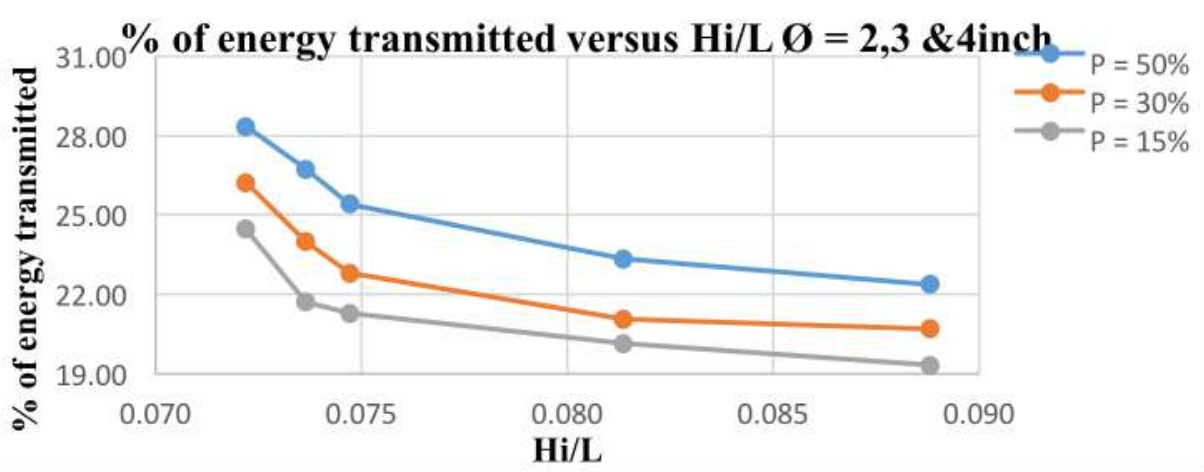

Fig. (18) \% of energy transmitted versus $\mathrm{Hi} / \mathrm{L}$ for $\emptyset=2.0,3.0 \& 4.0$ inch $\& P=(15,30 \& 50 \%)$

Comparison with El Saie Y.M. (2019), that he was tested the same models but in the solid state who's porosity equal to zero. Therefore, figures $(19,20,21,22 \& 23)$ shows the difference wave energy transmitted between the solid and porosities models.

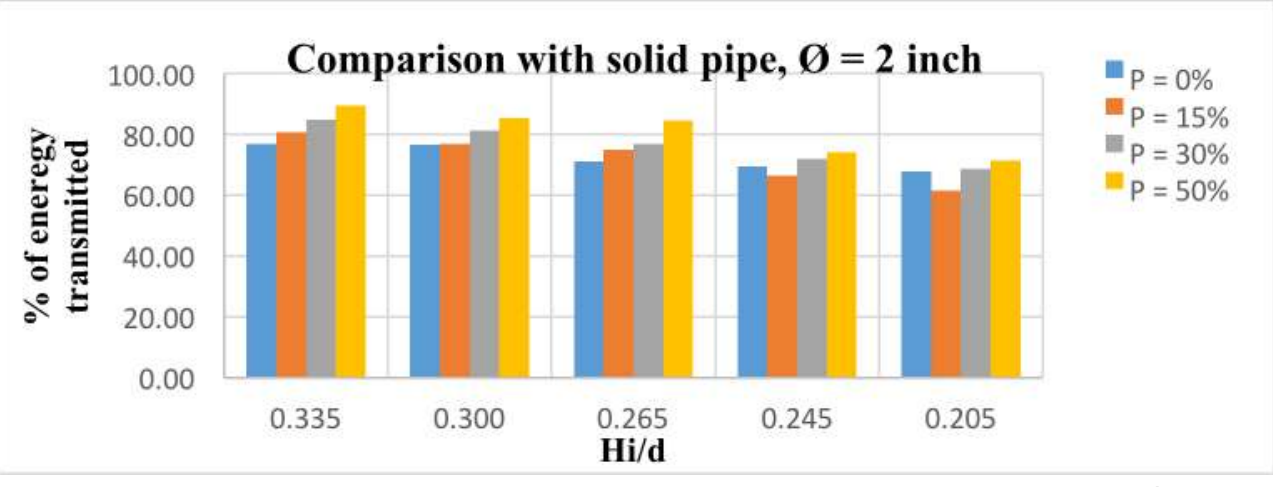

Fig. (19) Relation between \% of energy transmitted and $\mathrm{Hi} / \mathrm{d}$ for $\emptyset=2.0$ " 


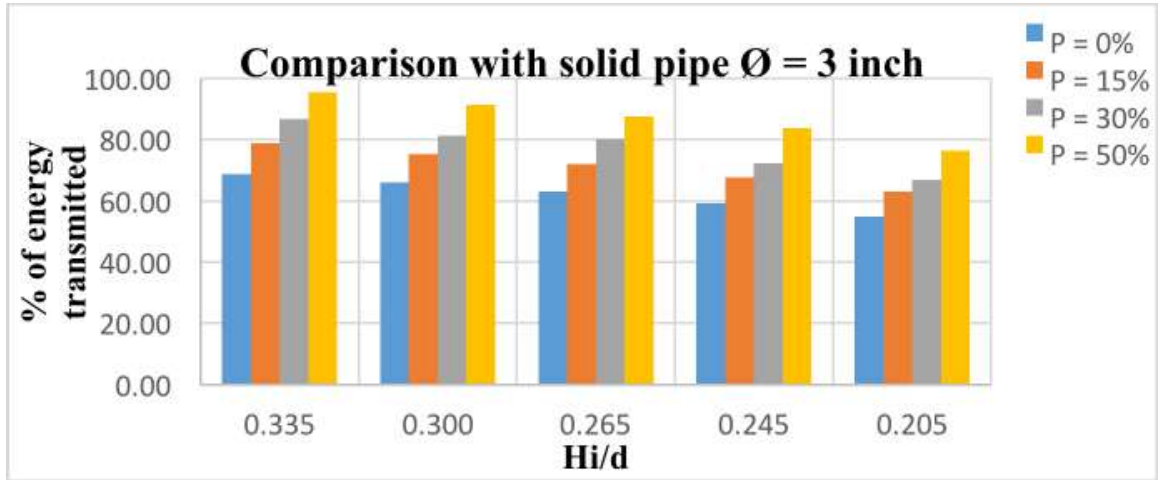

Fig. (20) Relation between \% of energy transmitted and $\mathrm{Hi} / \mathrm{d}$ for $\emptyset=3.0$ "

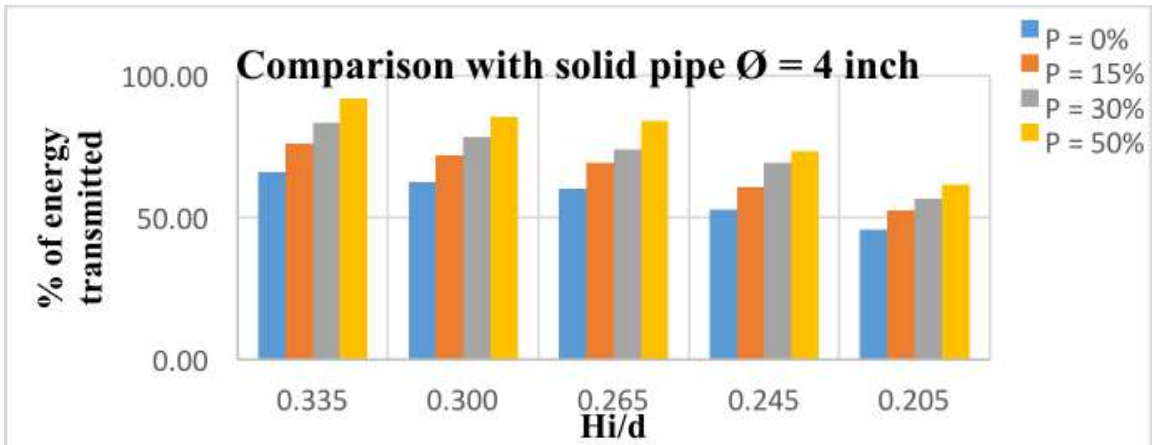

Fig. (21) Relation between \% of energy transmitted and $\mathrm{Hi} / \mathrm{d}$ for $\emptyset=4.0 "$

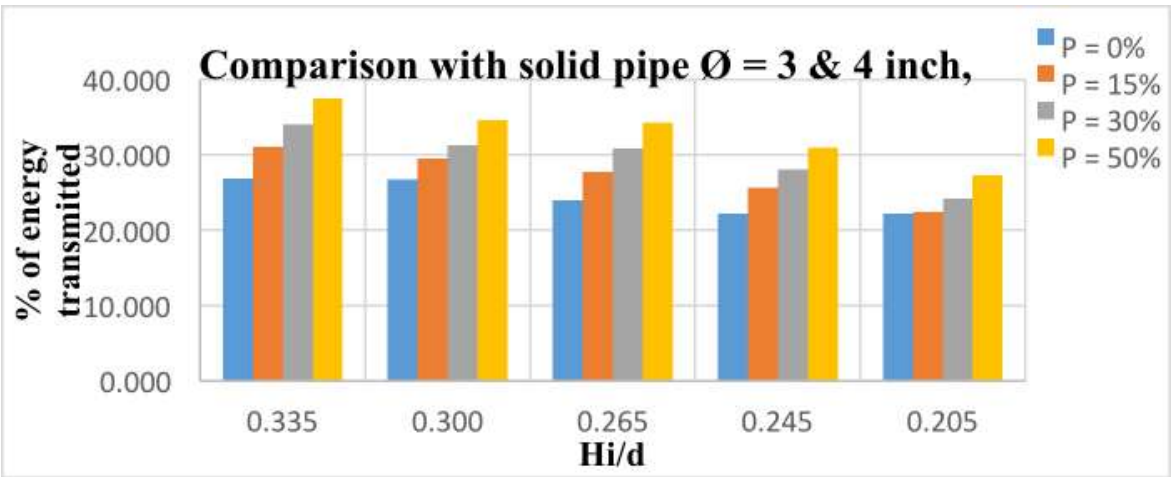

Fig. (22) Relation between \% of energy transmitted and $\mathrm{Hi} / \mathrm{d}$ for $\emptyset=3.0$ " \& 4.0"

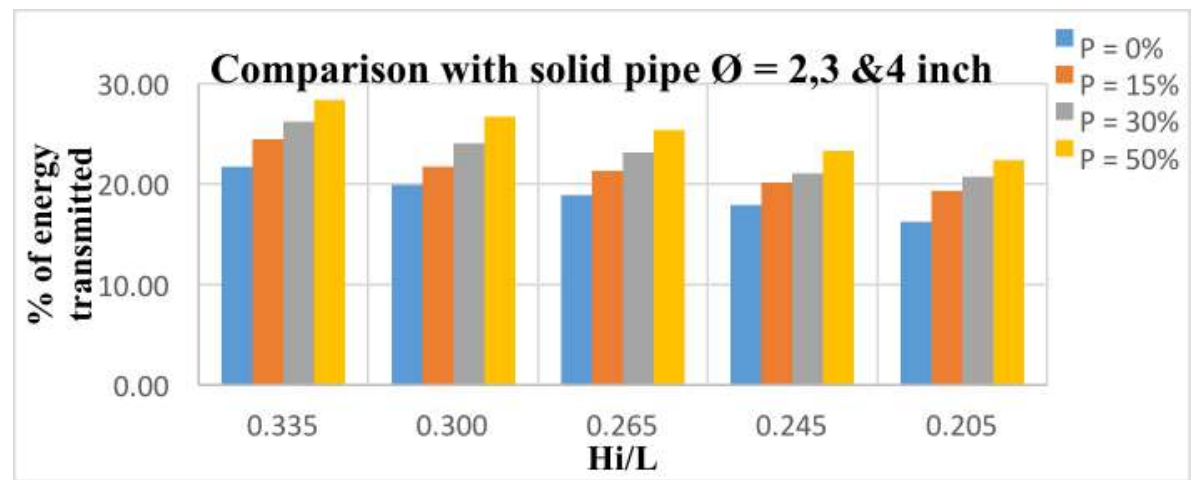

Fig. (23) Relation between \% of energy transmitted and $\mathrm{Hi} / \mathrm{d}$ for $\emptyset=2.0 ", 3.0 " \& 4$ "

\section{CONCLUSIONS}

The main conclusions of this paper are as follows:

- The coefficient of dissipation $\mathrm{Cd}$ is directly proportional with the diameter of the horizontal floating pours pipes, also the coefficient of transmitted $\mathrm{Ct}$ is inversely 
proportional with the diameter of the experimental floating pours models, with the difference wave steepness.

- The percentage of energy transmitted for the five models with $\mathrm{P}=(15 \%, 30 \& 50)$, and different wave steepness are approximately in average as follows:

* Model one ( $\varnothing=2.0$ inch), $(72,77.5$ \& $81.5 \%)$.

* Model two ( $\varnothing=3.0$ inch), (71, 76 \& $84 \%)$.

$*$ Model three $(\varnothing=4.0$ inch), $(63,70 \& 76 \%)$.

* Model four ( $\varnothing=3.0 \& 4.0$ inch), $(27,29.5$ \& $32 \%)$.

* Model five ( $\varnothing=2.0,3.0 \& 4.0$ inch), (21, $23 \& 26.5 \%)$.

- The difference of changing (Cd) between models $(1,2,3,4 \& 5)$ for porosities $(\mathrm{P}=15,30$ $\& 50$ ) for minimum wave steepness is nearly $20 \%$ and in maximum wave steepness is approximately $10 \%$.

- For comparison with hollow solid pipes with porosity equal to zero for the same five models with $\mathrm{P}=(15,30 \& 50 \%)$ and difference $\mathrm{Hi} / \mathrm{d}$ the difference of energy transmitted is as follows:

* For $\emptyset=2.0$ inch, $(5,11 \& 13 \%)$.

* For $\emptyset=3.0$ inch, $(9,14 \& 22 \%)$.

$*$ For $\emptyset=4.0$ inch, $(10,16 \& 24 \%)$.

* For $\emptyset=3.0 \& 4.0$ inch together, $(4,9 \& 12 \%)$

* For $\emptyset=2.0,3.0 \& 4.0$ inch together, $(3,5 \& 7 \%)$.

- Minimum porosity leading to maximum energy dissipation and minimum energy transmitted.

\section{References}

R. A. Dalrymple, M. A. Losada, and P. A. Martin, "Reflection and transmission from porous structures under oblique wave attack, "Journal of Fluid Mechanics, vol. 224, pp. 625-644, 1991.

Wang, H.Y., Sun, Z.C., 2010. Experimental study of a porous floating breakwater. Ocean Eng. 37, 520-527.

Garcia, N., Lara, J.L., Losada, I.J., 2004. 2-D numerical analysis of near-field flow at low crested permeable breakwaters. Coast Eng. 51, 10.

Jacobsen, N.G., van Gent, M.R.A., Wolters, G., 2015. Numerical analysis of the interaction of irregular waves with two dimensional permeable coastal structures. Coast Eng.102, 13-29.

Jensen, B., Jacobsen, N.G., Christensen, E.D., 2014. Investigations on the porous media equations and resistance coefficients for coastal structures. Coast Eng. 84, 56-72.

Losada, I.J., Lara, J.L., Christensen, E.D., Garcia, N., 2005. Modelling of velocity and turbulence fields around and within low-crested rubble-mound breakwaters. Coast Eng. 52, 887-913.

A. G. Abul-Azm, "Wave diffraction through submerged breakwaters, "Journal of Waterway, Port, Coastal and Ocean Eng., vol. 119, no. 6, 1993.

Erik Damgaard Christensen, Harry B. Bingham, Andreas Peter Skou Friis , Alexander Kruse Larsen and Karsten Lindegaard Jensen, "An experimental and numerical study of floating breakwaters". Journal of Coastal Engineering 137 (2018) 43-58.

M. Isaacson, D. Papps, and E. Mansard, "Oblique reflection characteristics of rubble-mound structures," Journal of Waterways, Port, Coastal, Ocean Eng., ASCE, vol. 122, no. 1, 1996.

N Zheng, X M Liu1, C P Chen1, Y P Jiang and C W Zhang, "Experimental study on the wave dissipation performance and mooring force of porous floating breakwater". 2018 International Conference on Civil and Hydraulic Engineering (Icon CHE 2018).

E. M. Heikal and A. A. Attar, "Effect of beach slope on the efficiency of submerged breakwaters," in Proc. 2nd Int. Conf., Mansoura Univ., Mansoura, Egypt, 1997.

S. W. Twu, C. C. Liu, and W. H. Hsu, "Wave damping characteristics of deeply submerged breakwaters," Journal of Waterway, Port, Coastal and Ocean Eng., vol. 127, no. 2, 2001. 
Zhichao Fang, Longfei Xiao, Yufeng Kou and Jun Li. "Experimental study of the wave-dissipating performance of a four-layer horizontal porous-plate breakwater, Ocean Engineering 151 (2018) 222-233.

D. G. Stamos and M. R. Hajj, "Reflection and Transmission of waves over submerged breakwaters," Journal of Engineering Mechanics, vol. 27, pp. 99-105, 2001.

D. G. Stamos, M. R. Hajj, and P. Demetri, "Performance of hemi cylindrical and rectangular submerged breakwaters," Journal of Ocean Engineering, vol. 30, iss. 6, 2003.

C. L. Ting, M. C. Lin, and C. Y. Cheng, "Porosity effects on non-breaking surface waves over permeable submerged breakwaters, "Journal of Coastal Engineering, vol. 50, iss. 4, 2004.

F. J. Sidek and M. Wahab, "The effects of porosity of submerged breakwater structures on non-breaking wave transformations, "Malaysian Journal of Civil Engineering, vol. 19, no.1, pp. 17-25, 2007.

Jian Dai, Chien Ming Wang, Tomoaki Utsunomiya and Wenhui Duan, "Review of recent research and developments on floating breakwaters". Ocean Engineering 158 (2018) 132-151.

S. A. Womera, "Experimental and numerical investigation on wave interaction with submerged breakwater," M.Sc. Engineering Thesis, Bangladesh University of Engineering and Technology, Dhaka, 2011.

C. Vahid and C. Fatemeh, "Experimental studies of wave transmission through single perforated sheets and upright perforated wave filters," Journal of the Persian Gulf, vol. 2, no. 5, 2011.

Y. Wu, S. Hsiao, and G. Chen, "Solitary wave interaction with a submerged permeable breakwater: Experimental and numerical modeling," in Proc. 33rd Conference on Coastal Engineering, Santander, Spain, 2012, mound breakwaters. CoastEng. 52, 887-913.

El Saie, Y. M., "Performance Analysis of a Floating Hollow Cylindrical Breakwater in Experimental Study." IOSR Journal of Mechanical and Civil Engineering (IOSR-JMCE), vol. 16, no. 3, 2019, pp. 01-07. 